\title{
The costs of affective disorders
}

\section{Second Workshop on Costs and Assessment in Psychiatry*}

\author{
Hugh Freeman, Editor, The British Journal of Psychiatry
}

Professor Alan Maynard from the University of York stated that most treatments for most episodes of psychiatric disorder are of unknown cost-effectiveness, while there is also evidence of gross under-treatment (e.g. depression) and inappropriate treatment (e.g. schizophrenia). Cost-of-illness studies are popular, but of limited use for numerous reasons, e.g. because disease definition is disputed, incidence and prevalence are poorly measured, many patients are poorly identified, and are under- or untreated. Secondly, they do not inform decision-makers (e.g. purchasers of health care) about the relevant margins in costs and benefits - information which is central to funding decisions. Much of the evaluation that is funded is narrowly focused, taking a myopic view of the relevant treatment options (all too often these are purely drug regimes, rather than competing packages of pharmaceutical and social care), and tending to ignore both the costs and benefits imposed on carers. These problems might be mitigated by identifying who wants what information to inform which decision, prohibiting clinical trials which ignore relevant costeffectiveness issues, agreeing standards of good practice in economic evaluation, which recognise both its strengths (e.g. explicitness) and weaknesses (e.g. lack of agreement about some aspects of evaluative practice), and ensuring that the design and execution are multidisciplinary.

Lehtinen et al examined factors which affect the treatment of depression and anxiety neurosis in an extensive epidemiological study of the Finnish adult population. The age-adjusted prevalence of depressive neurosis was $4.6 \%$, and that of anxiety or phobic neurosis $6.2 \%$; $32 \%$ of subjects with depressive neurosis and $26 \%$ of subjects with anxiety or phobic neurosis received some treatment for their disorder, more often by the primary care system than by the psychiatric specialist service. The simultaneous occurrence of a physical disease increased the probability of being treated for affective disorder.

In France, Souêtre investigated the impact of various antidepressant drugs (amitryptiline, clomipramine, fluoxetine) on the direct and indirect

*Held in Venice, 26-28 October 1992. costs related to depressive disorders. In $1,900 \mathrm{de}$ pressed patients, a positive correlation was found between severity of depression and the costs, as well as a positive correlation with the risk of work days lost. The number of days lost work was lower with fluoxetine than with conventional antidepressants.

Wyatt et al from NIMH reported that the price of medical care, as with most services, has increased over three-fold during the last 20 years. Treating chronic illnesses is a sizable portion of the total health care costs; in the United States, schizophrenia consumes 35-40 billion direct and indirect dollars. However, lithium is an example of an inexpensive treatment that brings chronic illness under control and has a major financial benefit; since 1969 , at least $\$ 12$ billion has been saved in direct hospital care costs through this treatment. Because patients who once required admission are now out-patients, a corresponding increase in out-patient costs might be expected. This has not occurred, however, because many of the new out-patients in time become stable. There have also been decreases in Supplemental Security Income benefits and Social Security Disability payments, so that the total direct cost savings have been over $\$ 13$ billion. Including the substantially increased productivity of treated patients, lithium has saved the United States over $\$ 40$ billion from 1969 to 1991. The most severe form of manic-depressive illness (Bipolar-I affects about one per cent of the population; it often strikes at a time when careers are just beginning and too often those who have a great deal to contribute. Only about one-third of these individuals are currently being treated in the USA.

From Sweden, Rutz et al described an educational programme on the diagnosis and treatment of depressive disorders to all GPs on the island of Gotland. Evaluations of it were made in 1985 and 1988: the short-term effects were decreased frequencies of sick-leaves and in-patient care. Prescriptions of antidepressant drugs increased, while prescriptions of major tranquilisers, sedatives, and hypnotics decreased, as did the frequency of suicide. Three years after finishing the educational programme, though, in-patient care had increased again and the suicidal rate returned almost to baseline values. The 
results seem to indicate that educational programmes have to be repeated about every second year, if long term effects are to be expected.

Bebbington reported that the economic impact of new medical treatments are likely to become increasingly important in the future. He and professor B. Jönsson had adopted two approaches. In the first, they used a top-down cost-of-illness methodology to arrive at a conservative estimate for the direct costs to the nation of depressive illness, giving an overview of its economic impact. In the second approach, a simulation model compared the cost of old and new antidepressant therapies. The older tricyclic antidepressants were represented by imipramine, while the comparison treatment was with paroxetine, a serotonin reuptake inhibitor. The results of the costeffectiveness model suggest that an expensive drug may in practice be the cheaper option, when compliance is poor with an inexpensive drug, since treatment failure is a major component of the cost of treatment. Natziandreu et al reported that recurrent depression results in significant costs to society and the individual in monetary and quality of life terms, but that these costs are often unrecognised. Recent studies have demonstrated that recurrent depression has a greater impact than other common debilitating conditions such as hypertension or arthritis. Maintenance treatment with sertraline has been shown to reduce the recurrence of depression effectively, but the economic effects of maintenance versus episodic treatment have yet to be examined. Maintenance treatment with sertraline resulted in improvement in patients' well-being and social functioning, as measured by "quality-adjusted life years' (QALYs), in comparison to episodic treatment. The cost of this improvement was approximately $\$ 4000$ (US) which is considerably less than, e.g. the chronic treatment of severe hypertension at $\$ 16,000$ (US) per QALY.

Paul Kind from the Centre for Health Economics, University of York stated that an understanding of the costs and benefits of existing forms of treatment is an essential requirement to the economic appraisal of new forms of therapy. Such comparative information is not always immediately to hand, since many factors combine to complicate the task of establishing these baseline costs, e.g. the limited availability of data in the public domain, and the underlying absence of useful measures of outcome. In response to these difficulties, a model has been developed which identifies the principal elements in management and treatment of depression.

\title{
Ninth International Psychiatric Conference of the Pakistan Psychiatric Society
}

\author{
M. FarRuKh HussaIn, Consultant Psychiatrist, St Augustine's Hospital, Canterbury, \\ Kent CT4 7LL; and M. AfZal JAVED, Consultant Psychiatrist, Mayo Hospital \\ Lahore, Pakistan
}

The Pakistan Psychiatric Society held its ninth international conference at the Pearl-Continental Hotel, Peshawar, from 8-10 December 1992. This biannual event was attended by more than 300 participants, 45 of whom were from abroad (including UK, USA, Australia, Germany, Switzerland, and Turkey).

The main theme of the conference was institutional $v$. community care. The conference debate was enlivened through a satellite linked with Professor Leonard Stein in Madison and Dr D. McGill (New York). Institutional care was proposed by Professor M. R. Chaudry (Lahore) and seconded by Dr Peter Hall (Worcester). The proposer for community care was Professor M. H. Mubbashar (Islamabad) and the seconder Dr Ian Pullen (Edinburgh). Professor I. A. K. Tareen and Professor Andrew Sims acted as discussants. The consensus was that the debate represented a false dichotomy and that both types of care were required and needed to be adequately funded.

A second important topic was Pakistan mental health legislation. The 1912 Lunacy Act (dating from the era of the British Raj) is still in force. Replacement legislation has been discussed over the past decade. A new draft Pakistan Mental Health Act 1992, similar to current British legislation, was discussed by a panel representing psychiatrists from the UK (Professor $R$. Bluglass and Dr M. Humphreys), Australia (Dr D. Greig) and Switzerland (DrV. Dittman). There was a vigorous discussion from the floor with a conclusion that the draft should be supported with certain important amendments.

The conference presented an unusual opportunity for psychiatrists from different cultures and health care systems to exchange views. It is hoped that the knowledge of Pakistan psychiatric services gained by those from the UK will help provide more appropriate training for trainees from Pakistan who take part in the Overseas Doctors Training Scheme.

Post conference tours to the Khyber Pass and places of historical interest were well supported and much appreciated by the foreign delegates.

The next International Conference of the Pakistan Psychiatric Society will take place in Lahore in December 1994. 